\title{
Fuzzy Control of Intersection Signal Based on Optimized Genetic Algorithm
}

\author{
Jie CAO, Yi WANG \\ College of Electrical and Information Engineering, Lanzhou University of Technology, China \\ 809860457@qq.com
}

\begin{abstract}
Keywords: Fuzzy control; Fitness; Evolutionary algorithm; Fuzzy control rules; Membership functions
\end{abstract}

Abstract. In order to adapt to the complex traffic and reduce the delay brought by simple traffic model, an improved adaptive genetic fuzzy control method was put forward for intersection traffic signal control. In order to improve the computational efficiency, the population size should be adjusted and the Gaussian membership function should be used. Dealing with local optimum happens in the early process of evolutionary algorithms, the method of increasing the part of the crossover and mutation probability has been used, so the stagnation of evolution process during high fitness individuals was avoid. To test the effectiveness and adaptability of this method, the classic method and the improved method was made to make the simulation respectively under high traffic flow, and the average delay data as a judge. The validation results show that the improved fuzzy control are effective in reducing the average vehicle delay in middle and later periods of the simulation. So that the efficiency of intersection traffic is increased.

\section{Introduction}

Fixed signal timing plan is adopted in early traffic control. It is difficult to establish accurate mathematical model under complex and enormous actual traffic scene. Most times are set on the basis of expert experience with worse robustness. Fuzzy control method enters traffic control field with new vitality. It does not require accurate model of controlled object, and can achieve excellent application effect under complex and random traffic scenes. Urban traffic control method based on fuzzy control theory still occupies a crucial position among many traffic intelligent control methods till present [1].

Pappis and Mamdani firstly proposed control method of urban unidirectional single intersection in 1976, and fuzzy control was applied for controlling single intersection signal lights [2] in the aspect of intersection signal light fuzzy control. Chen Hong, etc. improved on the experiment basis of Pappis. For example, two-stage structure control was adopted, and influence of non-critical traffic was considered [3]. However, these control methods were established on the basis of ideal intersection models. It was difficult to apply the methods in urban practical models. Then, Kim, etc. put forward classified intersection control method [4]. The control strategy can be timely adjusted according to traffic change, and different fuzzy control rules can be selected. However, the classified controller had coarser membership function and fuzzy control rule, which should be further improved. In literature $[5,6,7]$, fuzzy controller was further studied deeply, therefore fuzzy controller can form multi-level fuzzy controllers according to traffic condition and fuzzy rule. Then, scholars expanded research objects to line control, surface control and other control modes. Signal control methods of neighboring intersections or complicated traffic network were started. The stratified hierarchical method was utilized for adjusting phase difference or signal cycle among all intersections. Fuzzy controller was expanded to wider occasions $[8,9,10,11,12]$. In literature $[13,14]$, urban intersection control method with the adaptive strategy was proposed. The plan had the capacity of self-study and gradual optimization, and therefore prediction algorithm had undesired complexity and response time. 


\section{Problems in conventional fuzzy controller}

Cumbersome and fixed mathematical models are abandoned in fuzzy control method. It is an artificial intelligent control method for imitating human thinking, understanding and deduction mode to certain degree. Human knowledge, common sense, etc. are well expressed by natural language form so that input and output process model can be perfectly processed by computer. Various traffic conditions are handled and responded by the same method according to previous experience of obtaining traffic police command. Therefore, traffic control efficiency can be greatly improved. However, general fuzzy controller has common defects: firstly, there is no fixed and perfect mechanism for determining model and parameter during establishment of fuzzy control rule, which mostly depend on massive experiment data of professional personnel and design personnel. Suitable data is selected as final conclusion, and independent choices of designers are provided in the data. When the system model is larger, design process is complex, data has higher variability, and fuzzy rules can float in a large space. The section accuracy and rationality can not be controlled. Secondly, membership function of fuzzy variables is determined. Fuzzy controller performance is determined by membership function. However, determination of membership function belongs to a global optimization issue. Membership functions are different under many states. It is difficult to solve optimization of many functions currently. Thirdly, formulation of fuzzy rule and membership function means that they can not be rewritten again according to traffic flow change condition. The above problems can lower the effect of fuzzy control at different extents.

Conventional fuzzy controller has worse variability, wherein fuzzy control rule and membership function are designed by professional personnel in the aspect of signal control. The adopted parameters are also constant. Fuzzy control accuracy and rationality are greatly discounted under practical application circumstance of sharply changed traffic condition. Fuzzy adaptive control system based on genetic thought refers that adjustable variables are added in fuzzy rule related parameters and membership function related parameters according to basic principle of adaptive control. Genetic algorithm is combined with fuzzy control technique to form genetic fuzzy control system. Knowledge acquisition, knowledge representation, knowledge reasoning and decision making are organically combined mainly aiming at improving the design process of fuzzy controller, improving dynamic and static performance of fuzzy control system. Global search ability of genetic thought is mainly utilized for learning, improving and designing membership function related parameters and fuzzy control rule related parameters.

In the paper, optimization of fuzzy controller by applying improved genetic algorithm is discussed aiming at the condition of immutable fuzzy rule and immutable membership function parameters in conventional fuzzy control. The robustness of the whole control system can be improved, and therefore the control effect can be better improved under the condition of traffic flow change.

\section{Optimal design of genetic algorithm principle and algorithm}

Basic structure of genetic algorithm. Genetic algorithm is a search algorithm of generating and testing (Generate-and-Test) iterative operation. Its basic process can be divided into four parts, respectively including initialization, selection crossover and mutation.

Step 1: initialization; $m$ individuals with different chromosomes are randomly used. These individuals can jointly form initial generation groups.

Step 2: selection; difference in individual fitness is comprehensively considered. Individuals in pairs are randomly divided into $\mathrm{n}$ groups, and unpaired individuals are eliminated.

Step 3: crossover: corresponding new individuals are generated through crossover operation of through crossover operation of parent chromosomes in pairs according to the preset crossover method, crossover frequency and crossover probability. Individuals with higher fitness are generally selected according to different fitness degrees. Individuals with quantity of $\mathrm{m}$ are selected from two generations for retaining. Other individuals are eliminated [15]. 
Step 4: mutation; different mutation probabilities and mutation methods can be selected in different work tasks. Genetic factor assignment of each individual can be replaced by opposite genetic factor according to mutation probability and method. Then, the system can return to STEP2 for operation. Steps 2 to step 4 become an operating cycle in the genetic process, which is vividly called generation.

Genetic algorithm is regarded as a mass calculation method. All individuals in the group are regarded as objects. Selection, crossover and mutation are respectively three steps of genetic algorithm. They are also called main operators. Three operators are combined to form genetic operation. Five indispensable basic elements in genetic algorithm operation include the follows: coding of all parameters; determination of initial population; setting of fitness function; setting of genetic operation; determination of control parameters (mainly representing group size, various genetic operation probabilities, etc.)

Basic flow of genetic algorithm. All calculated quantities are rationally combined to form basic algorithm flow in genetic algorithm as shown in figure 1.

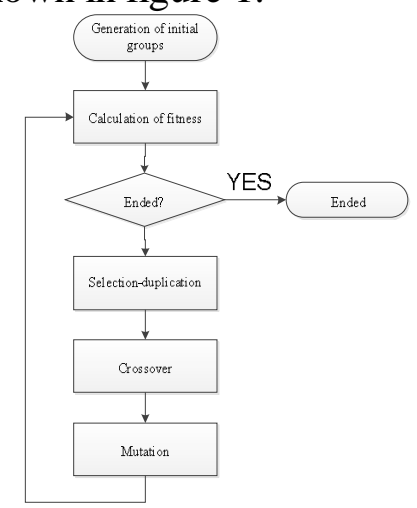

Figure 1 Basic flowchart of genetic algorithm

\section{Optimized fuzzy controller of genetic algorithm}

Optimization process. Fuzzy controller structure diagram optimized by genetic algorithm is shown in figure 2.

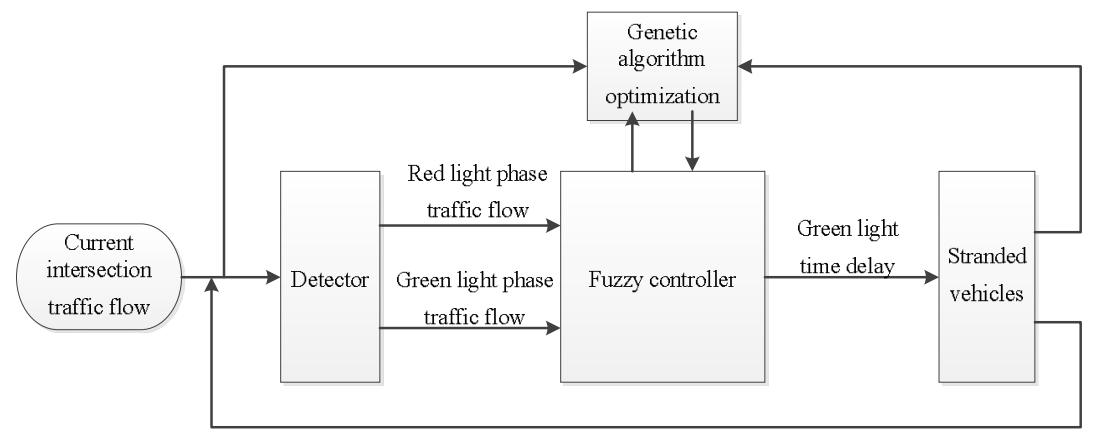

Figure 2 Block diagram of fuzzy controller optimized by genetic algorithm

\section{Encoding mode and generation of initial population}

1) Membership function

In the paper, Gaussian membership function is adopted as shown in formula 1. The characteristic curve is smooth:

$$
f(x, \delta, c)=e^{-\frac{(x-c)^{2}}{2 \delta^{2}}}
$$

There are two characteristic parameters and in the formula (1). Membership function shape is determined through selection. In the paper, initial value of fixed $\mathrm{c}$ is not changed. is optimized through genetic algorithm. The membership function shape is further optimized, thereby reducing chromosome length. There are parameters related to membership functions in input of each red-green phase and green light increase output: , and . After each parameter is amplified by one hundred times, eight-bit binary coding method is utilized. The value range $[1,255]$ corresponds to the value range 
$[0.01,2.55]$. The three parameters are jointly coded. The coding length is 3 multiplied by 8 , namely 24 bits. Corresponding values of parameters, and about membership function are 1.41, 0.9 and 0.7.

Population size is the factor that should be firstly determined in operation of genetic algorithm. Too small population has insufficient optimization space. The diversity of population can not be guaranteed, which is likely to lead to advanced convergence. In addition, too large population also can lead to too large search space. The search efficiency can be lowered. Therefore, too large population scale also can be selected. The best population selection scope is 20 to 30 aiming at conventional optimization problem.

\section{2) Fuzzy rule}

Because too long chromosomes are not beneficial for calculation during larger data size, some operations are required for improving calculation accuracy and speeding up the convergence speed. Real number coding is adopted for writing chromosomes corresponding to fuzzy rules. Each fuzzy set has seven membership functions. Each fuzzy set also corresponds to one pair of input-output variable one by one. Therefore, there are $7 * 7$ (namely 49) fuzzy rules in the chromosomes. Therefore, fuzzy variables are converted into numbers. One two-dimensional real number array can represent the whole fuzzy rule set (as shown in table 1). Next, two-dimensional real number array is converted into one row vector, thereby displaying the fuzzy rule. In the method, the first quantity of the next row can be inserted into the former row finally. In the algorithm, 49 optimal solutions are selected from all fuzzy control rules through operation. The 49 rules are basic basis of signal control.

Table 1 Coding list of fuzzy control principle

\begin{tabular}{|c|c|c|c|c|c|c|c|}
\hline $\mathrm{Q}_{\mathrm{r}}$ & HS & $\mathrm{S}$ & JS & $\begin{array}{l}\mathrm{Q} \\
\mathrm{C}\end{array}$ & $\mathrm{JL}$ & L & HL \\
\hline $\mathrm{VS}$ & 1 & 2 & 3 & 4 & 5 & 6 & 7 \\
\hline $\mathrm{S}$ & 1 & 2 & 3 & 4 & 5 & 6 & 7 \\
\hline SP & 1 & 2 & 3 & 4 & 5 & 6 & 7 \\
\hline $\mathrm{C}$ & 1 & 2 & 3 & 4 & 5 & 5 & 6 \\
\hline MP & 1 & 2 & 3 & 3 & 5 & 5 & 6 \\
\hline $\mathrm{M}$ & 1 & 1 & 3 & 2 & 3 & 4 & 5 \\
\hline VM & 1 & 1 & 3 & 2 & 3 & 3 & 4 \\
\hline
\end{tabular}

Design of fitness function. Fitness functions in genetic algorithms are mostly based on traffic optimization objective functions in general genetic algorithm, such as left-turning vehicle delay, average queue length, etc. The optimization objective function selected in the paper is average vehicle delay. Delay value is not negative necessarily as objective function under actual application situation. The fitness function itself is also non-negative. Since it is expected to obtain larger fitness value, the minimum objective function also can be obtained on the basis of practice. Therefore, values of objective function and fitness function are reversely changed. Namely, the obtained objective value is smaller, the fitness function value is naturally larger, and vice versa. Therefore, mapping relationship can be formed by fitness function and objective function. The optimization direction of objective function corresponds to fitness function increase direction in the formed mapping.

Wherein, $d$ is regarded as average vehicle delay, and it is objective function selected in the paper. D represents total delay of vehicles passed in y cycles.

$$
\left.D=\sum_{x=1}^{y}\left[\sum_{m=1}^{n}\left[\sum_{i=1}^{k}\left(Q_{i g}+\sum_{j=1}^{m} q_{i j}\right)\right]\right]+\sum_{m=1}^{g}\left[\sum_{i=1}^{k} Z_{i}\left(Q_{i r}+\sum_{j=1}^{m} q_{i j}-q_{s} m\right)\right]\right]
$$

In the formula, all parameters are shown as follows: qig is the vehicle quantity preserved after red light skipping of last cycle on the ith lane in red light phase of this cycle, qij is the quantity of vehicles which arrive in the intersection when the red light is sustained for $\mathrm{j}$ seconds on the ith lane at red light phase of this cycle, $\mathrm{k}$ refers to lane quantity at red light direction, qs is set as lane saturation, Qir is the waiting vehicle quantity after red light skipping at the ith lane during green light of this cycle, A refers to total arrival vehicle quantity at all directions during all operation time, and $\mathrm{d}$ refers to average delay time of each vehicle. Fitness function formula $\mathrm{F}$ is shown in formula 3.

$$
F=\frac{1}{d}=\frac{A}{D}
$$




\section{Genetic operation}

\section{1) Selection}

Selection according to fitness: individuals with high fitness can be selected with higher probability. Data and probability show that the probability of selecting individuals is proportional to its fitness value. It is set that $\mathrm{i}$ is available in the population with size Mi, its fitness is fi, so the probability formula of selecting $\mathrm{i}$ is shown as follows:

$$
P_{i}=\frac{f_{i}}{\sum_{i}^{M} f_{i}}
$$

Individual selection probability $\mathrm{Pi}$ is calculated according to formula (4). is produced following calculation. The value scope of is $[0,1], \mathrm{P} 1+\mathrm{P} 2+\ldots+\mathrm{Pi}-1<\mathrm{P} 1+\mathrm{P} 2+\mathrm{Pi}-1+\mathrm{Pi}$. Existing optimal individuals cannot be destroyed in order to get more optimal solution of population, and the optimal retention strategy is adopted under the condition. Two former individuals with the highest fitness are kept. Crossover process is exempted by the two individuals, which can directly enter population of next generation. In addition, two individuals with the lowest fitness are removed. Individuals with the highest fitness can be applied to generate new individuals with equal quantity in order to supplement population quantity and ensure quality.

\section{2) Crossover}

The $\mathrm{n}-4$ individuals are remained through the above operation processes. The individuals are randomly paired according to probability Pc. Individuals which are paired synchronously implement gene allelic exchange with corresponding individuals respectively during crossover process. Pc0 is still selected as crossover probability.

\section{3) Mutation}

Individuals retained in previous operation and newly generated individuals are ruled out. Each remaining individual can be mutated according to probability Pm. Individual gene is still mutated in the mutation process according to probability $\mathrm{Pm} 0$.

\section{Improved genetic algorithm}

Factors capable of controlling computing efficiency in genetic algorithm computing include crossover probability and mutation probability. It is possible to produce excellent gene structure damage or errors similar to gene crossover under the condition of improperly selecting Pc and Pm values. Similar conditions can lead to unhealthy and converged evolution process. Scholars develop genetic algorithm with adaptive function in order to prevent similar situation. When local optimum condition is displayed in all fitness degrees of populations, such adverse condition can be effectively eliminated by increase of Pc and Pm. Conversely, when fitness diffusion condition occurs, excellent effects can be exerted by suitably increasing Pc and Pm. Similarly, when fitness is higher than population fitness, and Pc and Pm are relatively smaller, the monomers are rationally maintained, which are transmitted into newly-generated population. When the individual fitness value is smaller than average value of population fitness, Pc and Pm are also large naturally, thereby benefiting elimination of such individuals. Therefore, the adaptive capacity of Pc and Pm can be beneficial for discarding the dross and keeping the finer part in the iteration algorithm. Finally, $\mathrm{Pc}$ and $\mathrm{Pm}$ values which are most beneficial for solving practical problems are finally adjusted.

The adaptive method is relatively reasonable in the late stage of population evolution. However, the method can easily guide the evolution result to local optimization at the initial stage of group evolution. The change is quite unbeneficial for application. Therefore, further improvements are generated. Crossover mutation probability can not be sticky through additionally increasing crossover mutation probability of many individuals with the maximum fitness in the population.

$$
p_{c}= \begin{cases}p_{c 1}-\frac{\left(\mathrm{p}_{c 1}-\mathrm{p}_{c 2}\right)\left(f^{\prime}-f_{\text {avg }}\right)}{f_{\text {max }}-f_{\text {avg }}}, & f^{\prime} \geq f_{\text {avg }} \\ P_{c 1} & , f<f_{\text {avg }}\end{cases}
$$




$$
p_{m}= \begin{cases}p_{m 1}-\frac{\left(\mathrm{p}_{m 1}-\mathrm{p}_{m 2}\right)\left(f^{\prime}-f_{\text {avg }}\right)}{f_{\text {max }}-f_{\text {avg }}}, & f^{\prime} \geq f_{\text {avg }} \\ P_{m 1} & , f<f_{\text {avg }}\end{cases}
$$

In the formula: favg represents overall average fitness of the first generation, fmax represents the highest overall fitness, $\mathrm{f}^{\star}$ represents higher fitness of two above-mentioned fitness with crossover possibility, and $f$ represents the value of fitness of single mutation individuals, wherein the crossover mutation parameter is $\mathrm{Pc} 1=0.9, \mathrm{Pc} 2=0.6, \mathrm{Pm} 1=0.1, \mathrm{Pm} 2=0.01$.

\section{Simulation analysis}

In the paper, MATLAB is adopted for programming. One multi-phase fuzzy control concept is adopted for constructing simulation, thereby verifying the optimization results accordingly. Another program design adopts optimized fuzzy controller in order to generate comparative experiment, wherein genetic algorithm is utilized for optimizing fuzzy control parameters. Concrete operation parameters are shown as follows: simulation time is 1 hour in order to reach higher light time cycle number, thereby eliminating the influence of coincidence on simulation. Green light phase is the starting phase of simulation operation. The vehicle generation rate on four vehicle inlet road sections is set as 4 vehicles/second. The population size of $\mathrm{N}=20$ is selected for optimized genetic algorithm, and the evolutional generation is 80 . The crossover probability of individuals and genes is respectively set as $\mathrm{Pc}=0.9, \mathrm{Pc} 0=0.4$. Similarly, corresponding mutation probability is set as $\mathrm{Pm}=0.1, \mathrm{Pm} 0=0.1$.

4.1 Results of membership function optimized by genetic algorithm

Relieving efficiency brought by traffic intelligent control optimization is the most urgent under the condition of larger traffic pressure. High traffic flow data is mostly adopted in previous traffic optimization simulation. Therefore, simulation experiment is done under data with higher traffic pressure.

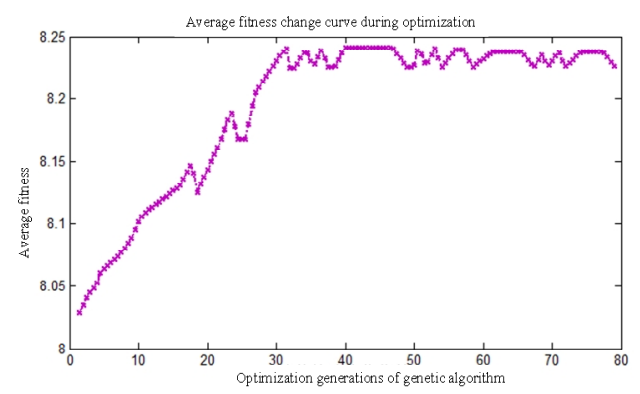

Figure 3 Average fitness curve of improved membership function

Figure 3 shows the fitness change curve of membership function after improvement. Improved parameters, and are 1.5, 0.8 and 1.1 .

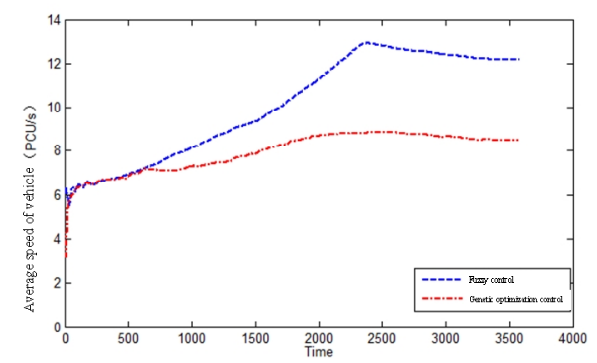

Figure 4 Vehicle average delay curve of improved membership function

Vehicles average delay curve is clearly shown in Figure 4 through simulation process for certain time. Two curves respectively represent classic fuzzy control and delay data after optimization. It is easy to distinguish the difference of two control means in the figure. Two control effects are similar within shorter time at the initial stage. Boundary of both curves is distinguished quickly after some fluctuations. Optimized fuzzy controller maintains vehicle delay under relatively stable growth extent. However, vehicle delay increase can not be better inhibited by classic fuzzy control. Fuzzy controller 
with optimized membership function obtains obvious advantages. The effectiveness of the optimization method is well proved in the simulation experiment till present.

Results of genetic algorithm optimization fuzzy rule. Fuzzy control method is utilized to complete control simulation on traffic flow on single intersection at the initial stage of simulation. Average capacity of the intersection should be settled after completion of the control cycle, which is the same as the membership function optimization method in the previous context. The settlement results are in line with evaluation value. The intersection fuzzy method can be improved by genetic ideas.

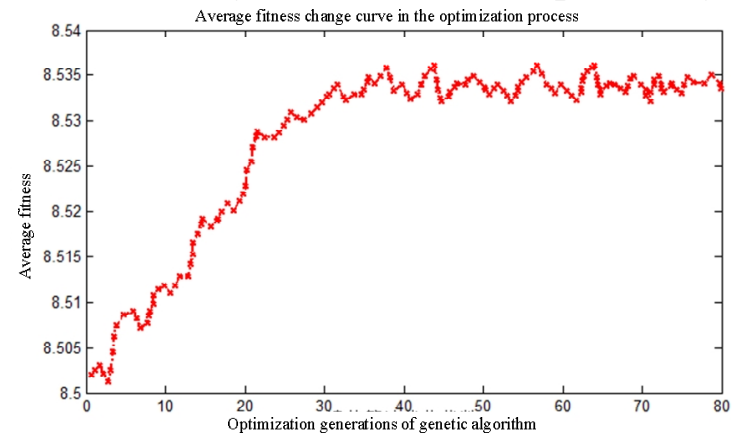

Figure 5 Improved fuzzy rule fitness curve

Table 2 Fuzzy rule after optimization

\begin{tabular}{|c|c|c|c|c|c|c|c|}
\hline \multirow{2}{*}{ Qr } & \multicolumn{7}{|c|}{$\mathrm{Qg}$} \\
\hline & HS & $\mathrm{S}$ & $\mathrm{JS}$ & $\mathrm{C}$ & $\mathrm{JL}$ & $\mathrm{L}$ & $\mathrm{HL}$ \\
\hline $\mathrm{VS}$ & HS & $S$ & JS & $\mathrm{JL}$ & $\mathrm{L}$ & $\mathrm{L}$ & $\mathrm{HL}$ \\
\hline$S$ & HS & $S$ & JS & $\mathrm{JL}$ & $\mathrm{JL}$ & $\mathrm{JL}$ & HL \\
\hline SP & HS & HS & $\mathrm{S}$ & $\mathrm{C}$ & $\mathrm{JL}$ & $\mathrm{JL}$ & HL \\
\hline C & HS & HS & $\mathrm{S}$ & $\mathrm{C}$ & $\mathrm{C}$ & JL & HL \\
\hline MP & HS & HS & $\mathrm{S}$ & JS & $\mathrm{C}$ & $\mathrm{JL}$ & HL \\
\hline $\mathrm{M}$ & HS & HS & $\mathrm{S}$ & JS & JS & $\mathrm{C}$ & $\mathrm{JL}$ \\
\hline VM & HS & HS & S & S & S & JS & $\mathrm{JL}$ \\
\hline
\end{tabular}

Delay curve of comparing fuzzy method of genetic thinking optimization and conventional fuzzy control method is shown in figure 6 . The simulation is still based on higher traffic flow. The fluctuation curve of average fitness function in the optimization process of fuzzy control rule is shown in figure 5. Table 2 shows the optimized fuzzy rule. Two simulation curves in figure 6 are respectively conventional fuzzy control method after expert experience learning and fuzzy control method optimized by genetic algorithm. Delay conditions obtained by different methods can be clearly observed from the curve in the figure. Since very rational expert experience is adopted in the paper, two control methods at the initial stage of curve change do not show larger gap. The curve change only has fluctuation is smaller scope. Therefore, the optimization effect is not prominent. However, the simulation time is postponed. Fuzzy controller with optimized genetic algorithm is adopted for embodying its advantage of adaptive change. The delay condition is prominently controlled in the simulation, which is slightly lowered compared with conventional method. In addition, it is assumed that the fuzzy rule selected in conventional fuzzy control method is not perfect enough, and the method adopted in the paper will embody stronger optimization effect in optimization simulation.

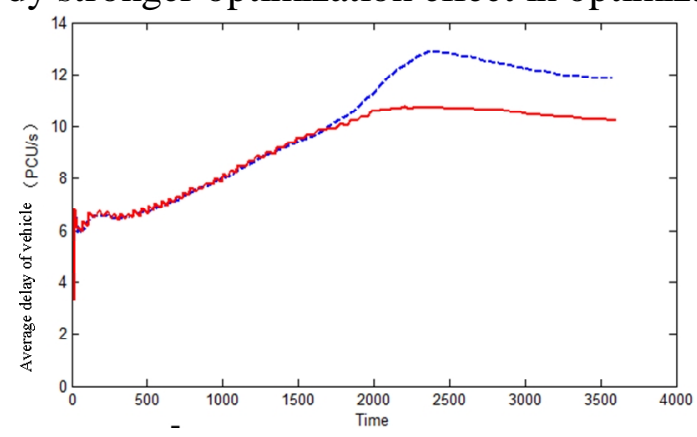

Figure 6 Vehicle delay curve of improved control rule 


\section{Conclusion}

Fuzzy control optimization problem under urban traffic single intersection condition is discussed in the paper. The delay phenomena incidence is lowered through increasing the crossover probability and mutation probability of individuals with high fitness. Suitable population size is selected during selection and treatment of population, which not only can guarantee population diversity and achieve sufficient optimization space, but also can improve search efficiency. Meanwhile, Gaussian membership function is adopted for making control characteristic more smooth, and improve the effect. In the paper, controller composition and optimization thoughts are described according to fact, and basic coding methods are also recorded. Fitness function selection and optimization improvement are further discussed. Simulation results show that the fusion optimization method proposed in the paper has better effect at the initial stage of operation. In addition, the whole control performance is further improved.

\section{References}

[1] Li Qunzu, Xia Qingguo, Ba Mingchun, Pan Wanpeng. President situation and development of urban traffic signal control system. Science Technology and Engineering, 2009, 9 (24):7436-7442.

[3] Chen Senfa, Chen Hong, Xu Jiqian. Two-stage fuzzy control and simulation thereof of urban single intersection traffic. Journal of System Simulation, 1998, 10 (2):35-40.

[4] Jongwan Kim.A Fuzzy logic Control Simulator for Adaptive Traffic Management. Proceedings of the Sixth IEEE International Conference on Fuzzy System,1997(3). 1533- 1539.

[5] Yunrui Bi,Jianming Li, Xiaobo Lu. Single Intersection Signal Control and Simulation Based on Fuzzy Logic. 2011 Third International Conference on Intelligent Human-Machine Systems and Cybernetics, 2011:87-89.

[6] C. V. Pham,W. L. Xu,J. Potgieter,F. Alain, F. C. Fang,W. L. Xu. A Probabilistic Fuzzy Logic Traffic Signal Control.19th International Conference on Mechatronics and Machine Vision in Practice, 2012:304-308.

[7] Jian Qiao,Naiding Yang, Jie Gao. Two-Stage Fuzzy Logic Controller for Signalized Intersection. IEEE Transactions on Systems, Man and Cybernetics, Part A: Systems and Humans, 2011,41(1):178-184.

[8] Rahman,R.Z.A.,Rhung,L.G.,Sarkan, H.M. Traffic signal control based on Adaptive Neural-Fuzzy Inference System applied to intersection. 2011 IEEE Conference on Open Systems (ICOS2011),Langkawi,Malaysia, 2011:231-236.

[9] Jee-HyongLee, HyungLee-Kwang. Distributed and Cooperative Fuzzy Controllers for Traffic Intersections Group. IEEE Transactions on Systems, Man, And Cybernetics Part C:Application and Reviews.1999.29(2):263-271.

[10]Lertworawanich Ponlathep. A simple adaptive signal control algorithm for isolated intersections using time-space diagrams. 13th International IEEE Annual Conference on Intelligent Transportation Systems, 2010:273-278.

[11] Mihai Hulea, Silviu Folea,Tiberiu Letia,George Mois. A collaborative approach to autonomous single intersection control. 19th Mediterranean Conference on Control and Automation,2011:694-699.

[12] Hawas Yaser E. A fuzzy-based system for incident detection in urban street networks. Transportation Research Part C: Emerging Technologies. 2007, 15(2): 69-95.

[13]J.J.Henry, J.L.Farges, J.L.Gallego. Neuro-fuzzy techniques For Traffic Control. Control Engineering Practice (1998):755-761. 
[14]C.Quek,M.Pasquier,B.Lim.A novel self-organizing fuzzy rule-based system for Modeling traffic flow behavior. Experts System With Application,2009.

[15] Chu Yongbin, Tang Zhen, Liu Xiaoping, Wei Xing, Zhang Li. Single-point traffic signal control method based on adaptive genetic algorithm. National 21st Computer Technology and Application Academic Conference-National 2nd Security Key Technology and Application Academic Conference, 2010:1-4.

[16] Wei Xing, Zhang Li, Wei Zhenchun, Zhai Yan. Research on traffic signal adaptive genetic control algorithm and its simulation. Journal of System Simulation, 2012, 24 (11): 2255-2258. 